\title{
TEE Provided Advantageous Information for a Surgical Resolution of Obstruction of SAS Suffering Difficulty in Removing CPB: A Case Report
}

\author{
Jin-ping Lin ${ }^{1}$, Cui juan Zheng², Wen-ying Zhou ${ }^{3}$ and Hai bo Song ${ }^{3 *}$ \\ ${ }^{1}$ Zhejiang University, China \\ ${ }^{2}$ The Affiliated Huai'an No.1 People's Hospital of Nanjing Medical University, China \\ ${ }^{3}$ Sichuan University, China
}

*Corresponding author: Hai bo Song, Department of Anesthesiology, West China Hospital, Sichuan University, Chengdu 610041, China

\section{ARTICLE INFO}

Received: 幽 November 08, 2019

Published: 幽 November 26, 2019

Citation: Jin-ping Lin, Cui juan Zheng, Wen-ying Zhou, Hai bo Song. TEE Provided Advantageous Information for a Surgical Resolution of Obstruction of SAS Suffering Difficulty in Removing CPB: A Case Report. Biomed J Sci \& Tech Res 23(2)-2019. BJSTR. MS.ID.003874.

Keywords: Supravalvular Aortic Stenosis (SAS); Transesophageal Echocardiography (TEE); Myocardial Contractility; Coronary Cusps

\section{ABSTRACT}

Congenital heart disease (CHD) is the most common type of congenital malformations. Congenital supravalvular aortic stenosis (SAS), one kind of CHDs, is strongly associated with abnormal aortic sac development with low prevalence while shows considerable impacts on infants' physical and mental development. Presently, surgical treatment is an effective therapy for SAS with acceptable surgical risk and good late survival. Evaluating the stenosis grade preoperative, the real-time status of heart during surgical correction are extremely crucial. Transesophageal echocardiography (TEE), has the advantages of clear images without interference of surgical field, is widely applied in diagnosis of cardiovascular diseases and intraoperative continuously monitoring. Here, we presented a successful case of surgical resolution of obstruction of congenital SAS in an infant who suffered difficulties in removing cardiopulmonary bypass (CPB) under the guidance of TEE.

Abbreviations: VC: Vena Cave; RA: Right Atrial; RV: Right Ventricular; PA: Pulmonary Artery; PV: Pulmonary Vein; LA: Left Atrial; AO: Aorta

\section{Introduction}

Congenital supravalvular aortic stenosis (SAS), characterized by congenital narrow of the ascending aorta, occurs sporadically and has an incidence of 1 in 20000 newborns [1]. Etiology is still obscure while various factors make contributes to SVAS such as genetic abnormalities including ELN gene [2], vitamin metabolism deficiency [3] and iatrogenic causes [4,5]. In 1961, Williams [6] firstly described the characteristics of congenital SAS such as mental retardation, macroscopic features which appears to originate from soft-tissue similarities while with little business of skulls, tortuous retinal vessels, lower weight for ages and the likes. However, therapy for SAS is still limited, and surgical approach for SAS repair is a preferred option currently [7]. Due to it is difficult to resolute the obstruction of SAS so that evaluating the stenosis grade preoperatively and the real-time heart function during the surgical correction are extremely essential for providing basis for perioperative team to work out the best therapeutic schedule. Presently, transesophageal echocardiography (TEE) is frequently used intraoperative for it's able to identify new pathologic findings, assess surgery procedure and potentially influence surgical decisions [8].

\section{Case Presentation}

A four-month-old male infant weighed $3.5 \mathrm{~kg}$ was admitted to our hospital due to cardiac soufflé was found two months ago. The patient presented with a graded $3 / 6$ systolic murmur without pal- 
pable thrill, developmental retardation and weight gained slows down refers to peer infants. Preoperative transthoracic echocardiography (TTE) confirmed it was narrow upper the aortic valve with the narrowest part was the sinus canal junction and the diameter was about only $4 \mathrm{~mm}$ with blood flow accelerated considerably (Fig- ure $1 \mathrm{~A}$ ), pulmonary value annulus diameter was about $7 \mathrm{~mm}$ with normal trunk and branch, pulmonary blood flow accelerated as well (Figure 1B). Other preoperative examinations showed no significant abnormalities. Then, surgical aortic and pulmonic stenosis correction under cardiopulmonary bypass (CPB) was planned.

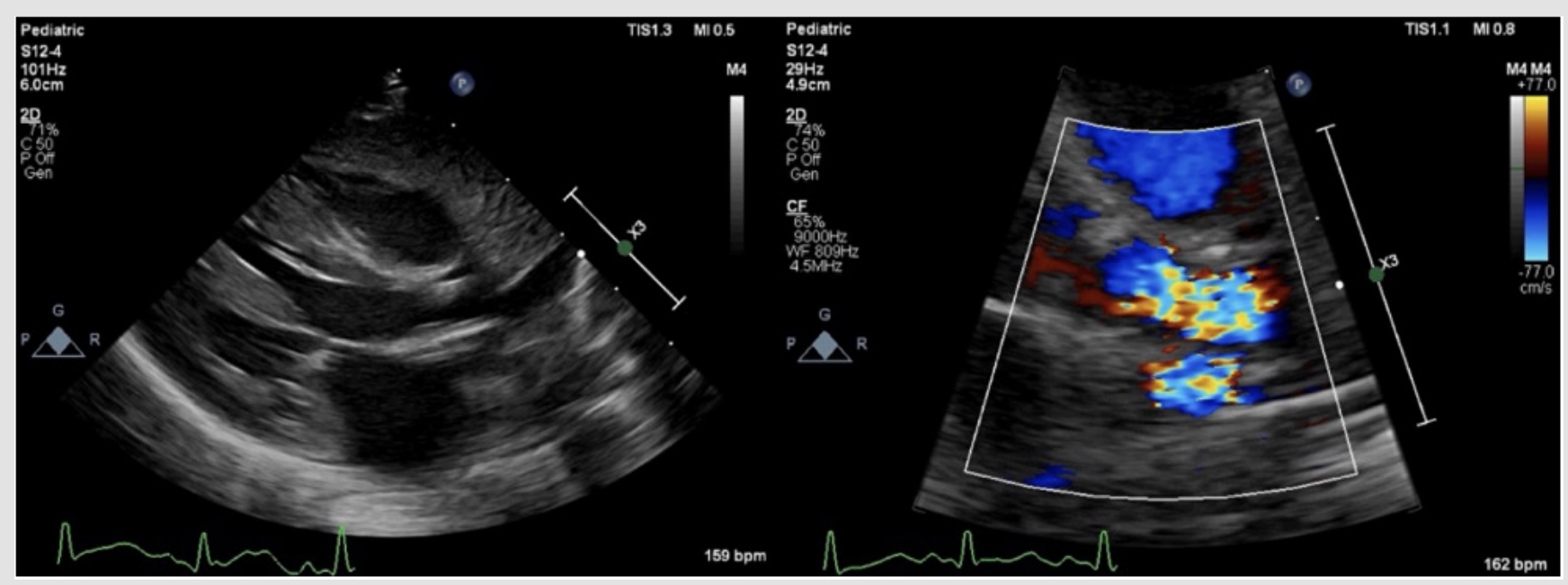

Figure 1A: Preoperative TTE. Preoperative TTE demonstrated it was narrow upper the aortic valve, and the narrowest part was the sinus canal junction with the diameter was about only 4 millimeter(mm), inner diameter of ascending aorta was about $8 \mathrm{~mm}$, the ventricular septum and posterior wall of left ventricle were thickened. The blood flow accelerated with the Vmax was $4.8 \mathrm{~m} / \mathrm{s}$ and PGmean was $57 \mathrm{mmHg}$.

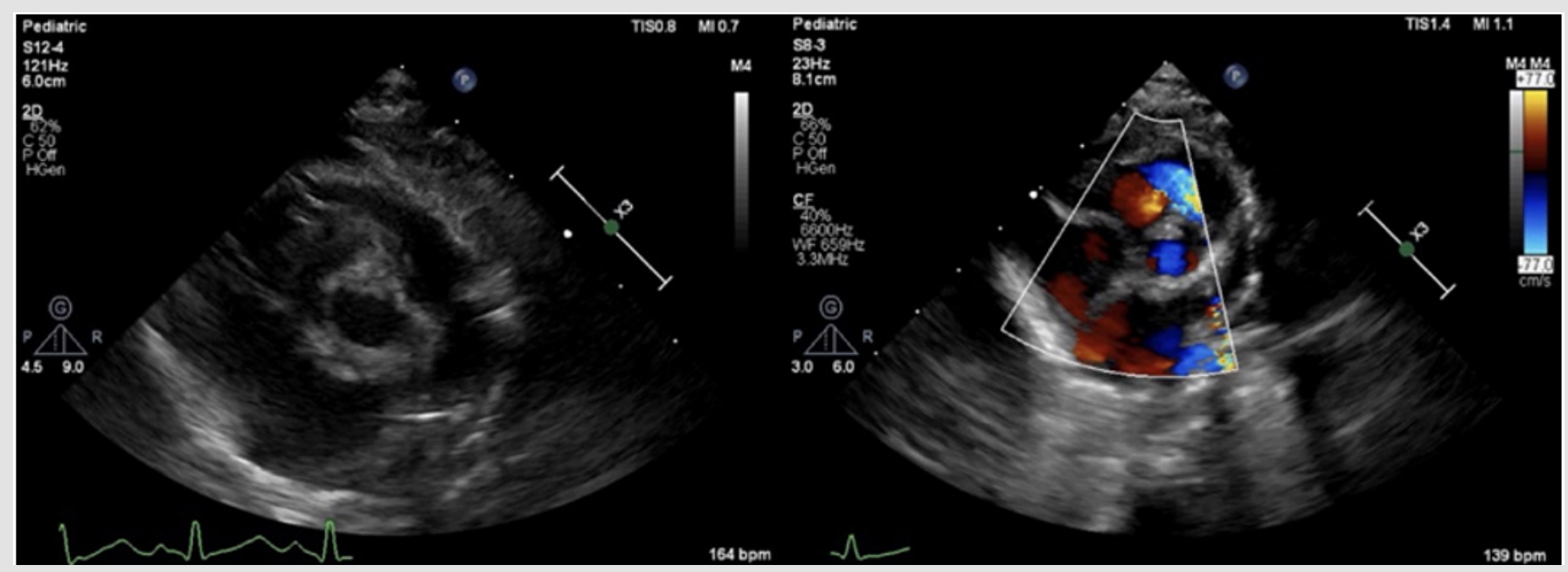

Figure 1B: Preoperative TTE. Pulmonary value annulus diameter was about $7 \mathrm{~mm}$ with normal trunk and branches. The blood flow accelerated with the Vmax was $3.3 \mathrm{~m} / \mathrm{s}$ and PG mean were $43 \mathrm{mmHg}$.

With standard anesthetic induction and tracheal intubation, evaluation by TEE (S8-3t/Pediatric, PHILIPS) was performed not only to reconfirm the location and degree of SAS (Figure 2A), but also for providing evidence to choose appropriate size/diameter of aortic cannulation. Median sternotomy was proceeded to expose the heart, CPB was achieved via the ascending aorta upper the narrowest location and the right atrium, and the surgical aortic and pulmonic stenosis correction went well. Unfortunately, the invasive blood pressure (IBP) was difficult to maintain without
CPB's support after heart resuscitation. Meanwhile, TEE showed the right heart functioned well. Contrarily, the left ventricular was enlarged coupled with shrinkage force was extremely weaken and mitral regurgitation (Figures 2B \& 2C). Minutes later, ventricular fibrillation occurred, and defibrillation was proceeded. CPB ran again, reluctantly and repeatedly. Surgeons advised anesthetists to pump norepinephrine $(2 \mathrm{ug} / \mathrm{kg} / \mathrm{min})$ intravenously to increase the cardiac after-load, unfortunately, this process leaded to much more difficulty in removing CPB. 


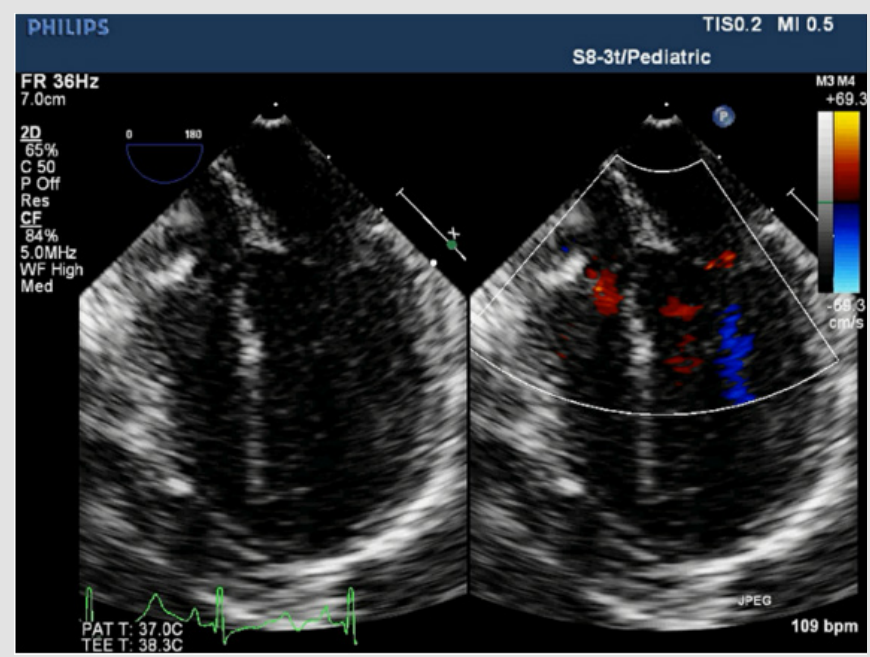

Figure 2A: Intraoperative TEE; Four-chamber view showed symmetry of ventricular size and the whole cardiac contractility was almost normal before surgery proceeded.

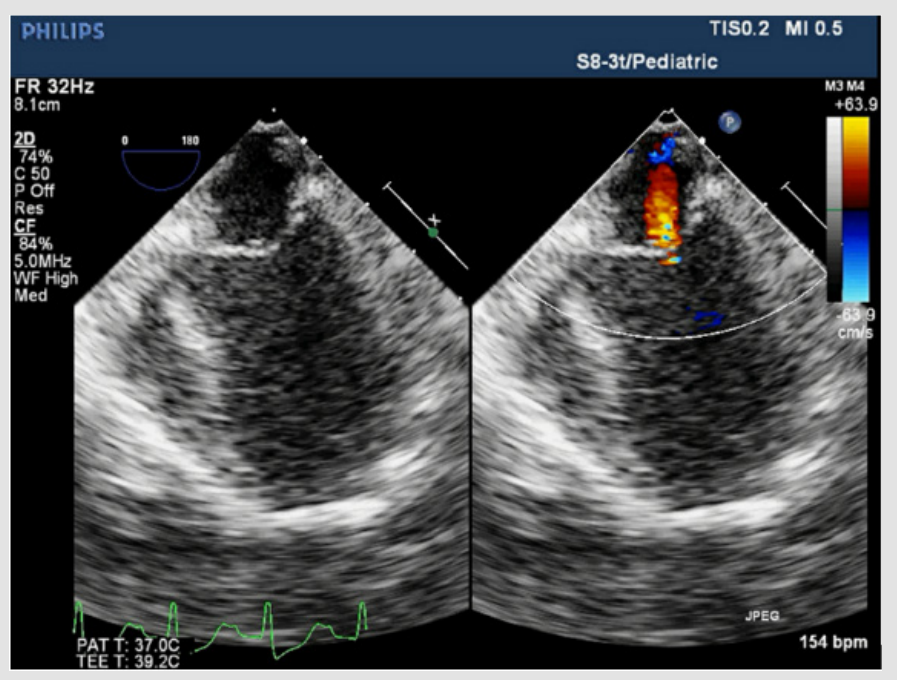

Figure 2B: Intraoperative TEE. Compared with preoperative values, TEE images showed the shrinkage force of left ventricular was weaken, coupled with mitral regurgitation. The left atrial and left ventricular was dilated seriously and the pressures measured by surgeons were $20 \mathrm{mmHg}$ and $12 \mathrm{mmHg}$, respectively.

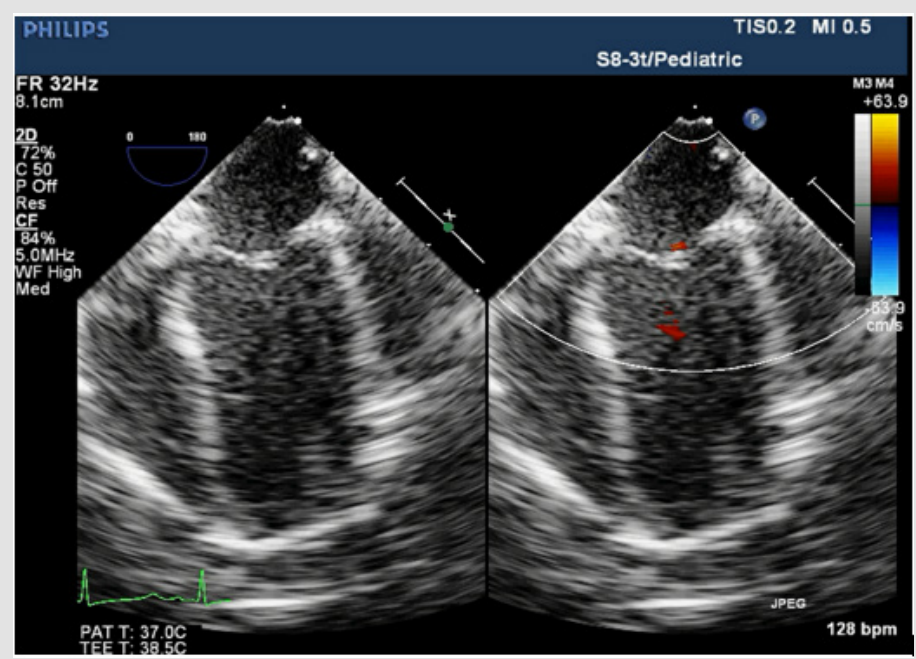

Figure 2C: Intraoperative TEE. The left ventricular myocardial contractility taken a turn for better, the shape of left ventricular was almost reached the normal level and mitral regurgitation alleviated after the surgeons reconstructed the left coronary cusp. 
According to TEE real-time images, combined with the "linear graph of angiocarpy" (a patent work from Hai-bo Song, patent number: 2015-L-00247942) (Figure 3), surgeons were suggested to check the cardiac apex's appearance, even coronary cusps and coronary arteries if the occasion should arise. The cardiac apex was wan without contractility! After open the aortic incision upper the non-coronary cusp decidedly for exploration, surgeons saw the coronary cusps were all abnormal deep and the non-coronary cusp after ascending aorta was broadened was relatively too large to compressed the left coronary cusp in ventricular diastole phase, which resulted in insufficient blood supplied to the left coronary artery generated dyspraxia and dilation of left ventricular related to mitral regurgitation(Figure 2B). Accordingly, surgeons broadened the left coronary cusp carefully using autologous pericardium. Finally, the left ventricular myocardial contractility taken a turn for better, the shape of left ventricular was almost reached the normal level preoperative and mitral regurgitation alleviated (Figure 2C). The whole CPB time was 286 minutes, ultrafiltration technology was used to wipe out inflammation factors. The baby patient suffered hemorrhage and pericardial tamponade in intensive care unit. After supportive treatments were given, the baby had an ideal recovery and discharged fifteen days later. Regular follow-up showed no abnormality.

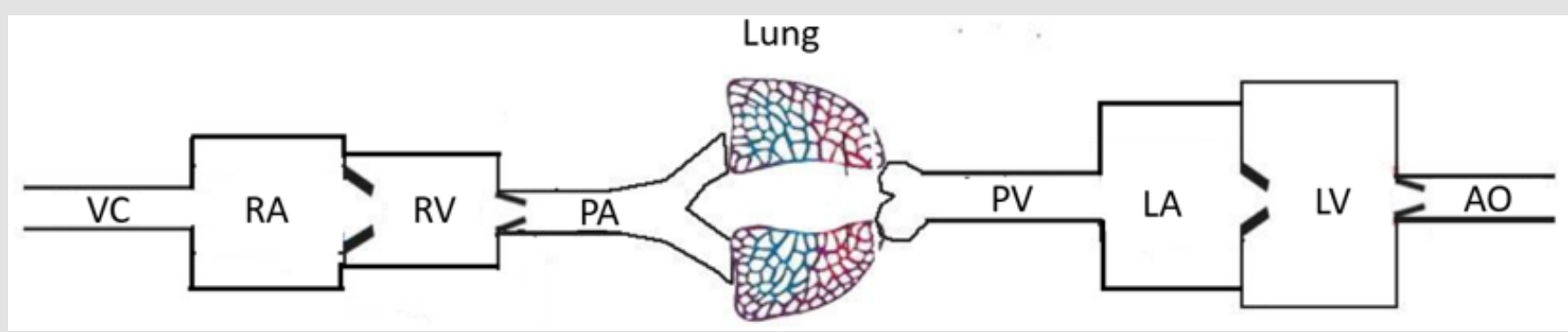

Figure 3: "Linear graph of angiocarpy". A patent work from Hai-bo Song (patent number: 2015-L-00247942). " $\rightarrow$ " in this figure meant the location of lesions and operative sites. After the obstruction of pulmonary artery corrected, the flow in pulmonary artery return to normal level and the right heart was functioning normally, while the left heart was not. According to this linear graph, combined with medical knowledge, it's largely because insufficient blood supply or left ventricular outflow tract obstruction when the left heart was dilated with weaken myocardial contractility.

\section{Discussion}

After Williams [6] firstly described the features of SAS in 1961 which are facial resemblance (the face are full, go with broad foreheads, set well apart eyes, heavy and dependent cheeks, wide mouths, and even pouting lips while chins are pointed), tortuous retinal vessels, physical and mental retardation, it has been gradually concerned. Later on, the association of SAS with characteristic facial features, oligophrenia and pulmonary artery stenosis was acknowledged as Williams-Beuren syndrome $[9,10]$. The etiology of congenial SAS is not quite clear and factors make contributes to it as we mentioned above. For acquired SAS, iatrogenic factor is perhaps the principal reason4, 5. Currently, therapy for SAS is still limited and surgical resolution including interventional procedure of the obstruction is a preferred option. However, kinds of surgical approaches for SAS repair have been proposed, while the optimal one is still controversial [7]. Either way, preoperational, intraoperative and postoperative evaluations of illness degree and heart function are significant essential.

As the improvement of monitoring conditions, TEE, offers better sound transmission and more windows which allows perioperative team to detect more valuable information compared with TTE, is widely used as a diagnostic and real-time monitoring tool [8]. The utility of intraoperative TEE is able to identify new pathologic findings and assess surgery procedure, consequently, influence surgi- cal decisions and provide the basis for perioperative team to make medicines, fluid managements, etc. In this case, intraoperative TEE images showed the right heart was favorable which meant surgical pulmonic stenosis correction was acceptable. For left heart, the conclusion that cardiac afterload was probably too low (resulted from SAS relieved) to maintain the IBP was deduced so perioperative team tried to increase the peripheral resistance by using vasoconstrictor while with ignorance of the information demonstrated by TEE images. TEE provided valuable information that the left ventricular was dilated and the myocardium was poorly contractile which meant increase the afterload blindly would only lead to more serious consequences. Later, combined TEE images with the "linear graph of angiocarpy" (Figure 3), perioperative team revalued there might be two possible causes leaded to this phenomenon. One was the size/diameter of aortic cannulation might be too big to block the left ventricular outflow tract, while according to the measurement by TTE\&TEE before the procedure, appropriate size and aortic cannulational location for intubation was selected. Another was there might be something wrong with coronary artery, especially the left coronary artery opening or trunk, might be abnormal or damaged during the operations, the closer to the coronary artery opening or trunk, the wider and more severe the left ventricular dysfunction is. The cardiac apex was wan without contractility and the exploration results after surgeons opened the aortic incision provided evidence for the later cause we raised. 
"The linear graph of angiocarpy", a comprehensive summary of circulatory system, is considerably helpful in learning cardiovascular knowledge and derivation of disease process. For instance, the pressure and volume of left atrial will increase with the possibility of left atrial appendage thrombus formed when patient suffering mitral stenosis while pressure and volume will decrease of the left ventricular. Moreover, the major clinical symtoms are dyspnea, hemoptysis generated from increased left atrial pressure and volume. In this case, the surgical pulmonic stenosis correction was successful so that the right heart was favorable without right ventricular enlargement or tricuspid regurgitation. For the left heart, both narrow outflow tract and insufficient coronary artery blood supply may result in dilation and insufficiency of left ventricular. We excluded the former according to preoperative TTE\&TEE measurement. The later was confirmed by surgeons. This linear graph of angiocarpy also can be applied in understanding pathophysiology of congenital heart disease including atrial septal defect (ASD), ventricular septal defect (VSD), anomalous pulmonary venous drainage, tetralogy of Fallot (TOF), etc.

There is something else we should pay enough focus on. It has been reported such multiple complications can occur when performing TEE in conscious adult patients as nausea, vomit, laryngismus, mucosal injury hemorrhage, arrhythmia, ventricular fibrillation and even death [11]. It's safer to perform intraoperative TEE with the incidence of complication ranges from $0.0 \%$ to $0.47 \%[12,13]$ and the main complication is postoperative dysphagia which exceeds 50\% [14]. Particularly, special attentions should be paid when we perform TEE examination in infant and young children. Not limited to gastrointestinal and circulatory complications, potential deleterious effects of compression of tracheobronchial structures and descending aorta by TEE probe in low-weight infants has been described as well. For total anomalous pulmonary venous connection (TAPVC) and other similar CHD, it's optimal to insert the TEE probe after the chest has been opened to avoid anomalous pulmonary venous being compressed [15]. Additionally, there is a consensus related to TEE procedure that the weight should equal to or greater than $3.5 \mathrm{~kg}$ and try not to perform transgastric views because the stomach wall is so thin that tend to bleed. For all patients, operating tenderly under proper safety conditions is compulsory. In short, the skills of clinicians are vital.

\section{Conclusion}

In this case, we considered that TEE is an advisable tool in surgical aortic stenosis correction which provided continuous guidance relevant with ventricular morphology and systole. Furthermore, a rare intraoperative complication during the resolution of obstruction of congenital SAS that the non-coronary cusp after ascending aorta was broadened was relatively too large to compressed the left coronary cusp in ventricular diastole phase resulted in insufficient blood supplied to the left coronary artery generated dyspraxia and dilation of left ventricular related to mitral regurgitation was introduced. Unfortunately, and sorrowfully, there were something we perioperative team didn't do well enough for this baby, for instance, the use of noradrenaline regardless of the left heart situation leaded to longer CPB time and more prolonged recovery. It's still a long way to go and a big challenge to be a qualified perioperative team member with cardiovascular knowledge and TEE skills.

\section{Acknowledgement}

Funding: This work was supported by research grants from the 1.3.5 project for disciplines of excellence, West China Hospital, Sichuan University (Zy2016101).

\section{Conflicts of Interest}

The authors have no conflicts of interest to declare.

Informed Consent: Written informed consent was obtained from the baby's guardian for publication of this manuscript and any accompanying images.

\section{References}

1. Micale L, Turturo MG, Fusco C, Augello B, Jurado LA, et al. (2010) Identification and characterization of seven novel mutations of elastin gene in a cohort of patients affected by supravalvular aortic stenosis. Eur J Hum Genet 18 (3): 317-323.

2. Metcalfe K, Rucka AK, Smoot L, Hofstadler G, Tuzler G, et al. (2000) Elastin: mutational spectrum in supravalvular aortic stenosis. Eur J Hum Genet 8 (12): 955-963.

3. Kitagawa H, Fujiki R, Yoshimura K, Oya H, Kato S (2011) Williams syndrome is an epigenome-regulator disease. Endocr J 58(2): 77-85.

4. Williams WG, Mathieu J, Culham G, Trusler GA, Olley PM (1979) Acquired supravalvular aortic stenosis. Ann Thorac Surg;27(4): 335-339.

5. Lee JH, Nam K, Park YH, Kim JT (2015) Iatrogenic supravalvular aortic stenosis detected by transesophageal echocardiography in a pediatric patient undergoing cardiac surgery. Anesth Analg 120(1): 26-29.

6. Williams JC, Barratt Boyes BG, Lowe JB (1961) Supravalvular aortic stenosis. Circulation 24: 1311-1318.

7. Padalino MA, Frigo AC, Comisso M, Kostolny M, Omeje I, et al. (2017). Early and late outcomes after surgical repair of congenital supravalvular aortic stenosis: a European Congenital Heart Surgeons Association multicentric study. Eur J Cardiothorac Surg 52(4): 789-797.

8. Han X, Song H, Zhou L, Chunling Jiang (2017) Surgical resection of right ventricular rhabdomyoma under the guidance of transesophageal echocardiography on a beating heart. Journal of thoracic disease $9(3)$ : E215-E218.

9. Ewart AK, Morris CA, Atkinson D, Jin W, Sternes K, et al. (1993). Hemizygosity at the elastin locus in a developmental disorder, Williams syndrome. Nat Genet 5(1): 11-16.

10. Micale L, Fusco C, Augello B, Napolitano LM, Dermitzakis ET, et al. (2008) Williams-Beuren syndrome TRIM50 encodes an E3 ubiquitin ligase. Eur J Hum Genet 16(9): 1038-1049.

11. Daniel WG, Erbel R, Kasper W, Visser CA, Engberding R, et al. (1991) Safety of transesophageal echocardiography. A multicenter survey of 10,419 examinations. Circulation 83(3): 817-821.

12. Chan KL, Cohen GI, Sochowski RA, Baird MG (1991) Complications of transesophageal echocardiography in ambulatory adult patients: analysis of 1500 consecutive examinations. J Am Soc Echocardiogr 4(6): 577-582.

13. Mishra M, Chauhan R, Sharma KK, Dhar A, Bhise M, et al. (1998). Realtime intraoperative transesophageal echocardiography--how useful? Experience of 5,016 cases. J Cardiothorac Vasc Anesth 12(6): 625-632. 
14. Eltzschig HK, Rosenberger P, Loffler M, Fox JA, Aranki SF, et al(2008) Impact of intraoperative transesophageal echocardiography on surgical decisions in 12,566 patients undergoing cardiac surgery. Ann Thorac Surg 85(3): 845-852

\section{ISSN: 2574-1241}

DOI: 10.26717/BJSTR.2019.23.003874

Hai bo Song. Biomed J Sci \& Tech Res

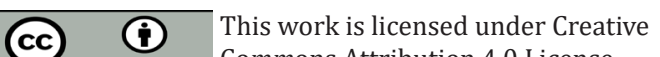

Submission Link: https://biomedres.us/submit-manuscript.php
15. Chang YY, Chang CI, Wang MJ, Lin SM, Chen YS, et al (2005) The safe use of intraoperative transesophageal echocardiography in the management of total anomalous pulmonary venous connection in newborns and infants: a case series. Paediatr Anaesth 15(11): 939-943.

$\begin{array}{ll}\text { BIOMEDICAL } & \text { Assets of Publishing with us } \\ \text { RESEARCHES } & \text { - Global archiving of articles } \\ \text { - Immediate, unrestricted online access } & \text { - Rigorous Peer Review Process } \\ & \text { - Authors Retain Copyrights } \\ & \end{array}$

